\title{
Bluetooth Broadcasting Performance: Reliability and Throughput ${ }^{\star}$
}

\author{
Kaan Dogan ${ }^{1}$, Guray Gurel ${ }^{1}$, A. Kerim Kamci ${ }^{1}$, and Ibrahim Korpeoglu ${ }^{2}$ \\ ${ }^{1}$ Department of Electrical and Electronics Engineering, \\ Bilkent University, Turkey \\ \{kaan, guray, kamci\}@ee.bilkent.edu.tr \\ ${ }^{2}$ Department of Computer Engineering, Bilkent University, Turkey \\ $\{$ korpe\}@cs.bilkent.edu.tr
}

\begin{abstract}
This paper studies the performance of Bluetooth broadcasting scheme. The transmission of a Bluetooth broadcast packet is repeated several times to increase the reliability of broadcast. We have analyzed the effects of baseband ACL packet type preference, on the broadcast performance in terms of reliability and effective throughput, over different channel characteristics (i.e. bit error rate). As the result of our analysis, we determined the optimal packet type and re-transmission count combinations that can provide the highest effective throughput values for various practical BER ranges. These results can be used at Bluetooth baseband layer to dynamically adapt to varying channel conditions and to achieve a good broadcast performance.
\end{abstract}

\section{Introduction}

Bluetooth (BT) standard [1] 2] includes a reliable broadcasting mechanism for applications that require data packets to be sent to more than one node in a BT piconet. Broadcasting is more energy and bandwidth efficient than separately unicasting the data to several nodes.

As broadcast packets are not acknowledged, reliability in BT broadcasting is achieved by simply repeating the transmission of the same packet $N_{B C}$ times. This causes, however, a tradeoff to be made between reliability and efficient channel utilization (high effective throughput).

In a BT piconet, the master node learns slaves' desired repetition count, $N_{B C}$, and announces the new value using the LMP_quality_of_service and LMP_ quality_ of_service_req messages [2. The master node can also determine an appropriate packet type to be used in broadcasting, since each packet type has different maximum size and error protection capability (different FEC coding). Broadcasting uses ACL (asynchronous connectionless link) packets and there are several types of ACL packets [2] 3. Hence it is possible for the master node to select the best combination for the packet type and repetition count in order to make a good tradeoff between reliability and throughput.

\footnotetext{
* This work is supported in part by The Scientific and Technical Research Council of Turkey (TUBITAK) under projects EEEAG-103E014 and EEEAG-104E028.
} 
In this work, we have investigated the optimum values for packet types and retransmission counts in terms of their effects on channel utilization under different bit error rates with a custom simulator. As far as we know this is the first paper that analyzes the BT broadcasting scheme in terms of throughput and reliability. In the next section we describe our methodology and simulation model. Then we provide our results and discussions.

\section{Methodology and Simulation Model}

A master node can acquire feedback about the channel condition and about the efficiency of broadcasting using the BT link manager messages. After broadcasting a certain number of packets to slaves, the master can poll the slaves so that the slaves can inform the master about the $N_{B C}$ values they want the master to use. The desired $N_{B C}$ value can be computed by a slave based on the quality of the reception and this value corresponds to a BER range. In order to ensure a minimum reliability level for all slaves, largest $N_{B C}$ feedback should be taken into account. Then, the master can choose the most appropriate packet type and repetition count based on these offline calculations and the desired $N_{B C}$ feedback from the slaves. This can be achieved without modifying the broadcasting mechanism defined in the BT specification [2.

A BT baseband packet consists of mainly three parts: an access code, a packet header, and the payload. The header is protected by $1 / 3 \mathrm{FEC}$ with whitening, providing an aggregate code distance of $d_{0}=6$, so the code is capable of correcting all single and detecting all quadruple errors [4. The access code is assumed to be protected by pseudo-random noise along with the expurgated code, which provides large Hamming distances $\left(d_{\min }=14\right)$. The trailer is a 4 bit pattern of either 1010 or 0101, and is used for DC compensation. Thus, the errors on access code and trailer are going to be ignored in the simulator [5].

$\mathrm{DH}$ packet payloads do not employ any protection scheme and error detection is achieved using CRC bits added to the end of the packets. Payloads of DM packets are protected by $2 / 3$ FEC (10/15 shortened Hamming Code), i.e. it can correct all single bit and detect all double bit errors in a 15 bit block.

The analytical approach discussed in 6] clearly reflects the BER and Packet Loss Rate (PLR) relationship. For packet types without FEC protection, the packet loss rate $p$ is related to $B E R$ and payload size $s$ (in bits) with the formula

$$
p=1-(1-B E R)^{s}
$$

For packets with 2/3 FEC protection, 15 bits are used to encode 10 bits of data, and this can correct one bit in every 15 bits. So the packet loss rate $p$ becomes:

$$
p=1-\left((1-B E R)^{15}+15 \times B E R \times(1-B E R)^{14}\right)^{s / 15}
$$

In our simulations, when the packet type is specified, our simulator calculates PLR from BER and payload size. During the simulations, number of packets 
lost and the amount of data successfully transported are recorded. These values are then used to calculate effective throughput (goodput) and reliability. Effective throughput is expected to decrease when the $N_{B C}$ value increases, since several packets occupying the channel contain the same data. Using large packets increases throughput, on the other hand using FEC decreases the effective throughput due to redundant bits inserted into packets. We define reliability as the ratio of correctly received packets to the packets transmitted. Various applications may require different degrees of reliability in terms of correct delivery of baseband broadcast packets to slaves from a master. Increasing $N_{B C}$ or using FEC protection increases reliability. In contrast enlarging packet payload, increases PLR and therefore reduces reliability.

We performed simulations to find out the repetition counts and packet types that will yield the maximum throughput for a given reliability range.

\section{Simulation Results}

We simulated the BT broadcast mechanism for various practical RF channel BER values. For each BER value that is fixed, we have evaluated the performance of each of the BT baseband packet types in terms of reliability and throughput. The $95 \%$ confidence interval of the simulation results are very tight and they are not discernible from the graphs. In the presented results, we have only depicted confidence mean values that are obtained from batches of 10 independent simulation runs in which the master sends $5 \mathrm{MBs}$ worth of broadcast data.

Performance of packet types under various BER values is closely related to each packet's vulnerability to error, which depends on packet payload size and the FEC scheme employed. In order to present the effect of $N_{B C}$ on reliability more clearly, we have simulated the broadcasting scenario for a fixed BER of $10^{-3}$. Figure 1 shows that relatively small values of $N_{B C}$ is sufficient for DM packets to provide reliable transmission. On the other hand, lack of FEC protection causes DH packets to exhibit poor performance. Finally, using packets with larger payloads reduces reliability, whereas increasing $N_{B C}$ yields more reliable broadcasts. When a desired level of reliability is specified, it is possible to find optimal packet types and $N_{B C}$ values that satisfy this constraint while providing maximum achievable throughput. Figure 2 show the maximum throughput obtained by ACL packet types under reliability constraints of $99.9 \%$. As BER is increased, $N_{B C}$ value is increased to satisfy reliability constraint and consequently maximum throughput decreases. The packet type that exhibits the best throughput performance changes over the practical BER range. Optimal repetition count and packet selection for $99.9 \%$ reliability is tabulated in Table 1 .

Having received the desired $N_{B C}$ values from the slaves, piconet master can find the corresponding BER according to the packet type being used in broadcast. The master can look up for optimal packet type and $N_{B C}$ value in Table 1 using this BER value. 


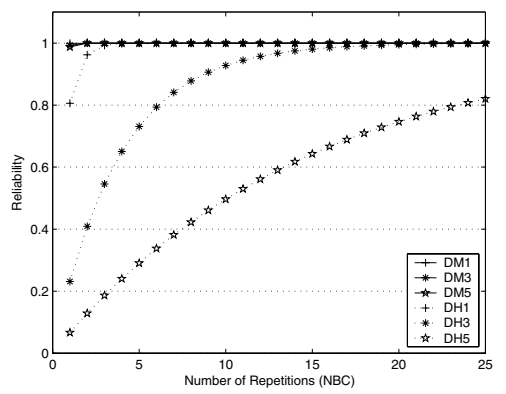

Fig. 1. Reliability vs $N_{B C}$ for BER of $10^{-3}$

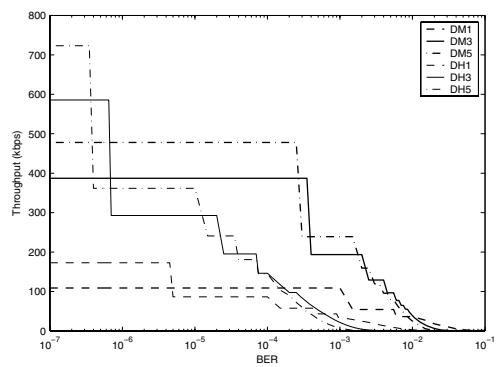

Fig. 2. Max. throughput for $99.9 \%$
Table 1. Optimum packet types \& $N_{B C}$ values for $99.9 \%$ reliability in all BER range

\begin{tabular}{|c|c|c|c|}
\hline Bit Error Rate & $\begin{array}{c}\text { Packet } \\
\text { Type }\end{array}$ & $\begin{array}{c}\mathrm{N} \\
B C\end{array}$ & $\begin{array}{c}\mathrm{T} \\
(\mathrm{kbps})\end{array}$ \\
\hline$\left[\mathrm{BER}<7.5 \times 10^{-7}\right]$ & DH5 & 1 & 723.00 \\
\hline$\left[7.5 \times 10^{-7}, 10^{-6}\right]$ & DH3 & 1 & 585.60 \\
\hline$\left[10^{-6}, 10^{-4}\right]$ & DM5 & 1 & 477.80 \\
\hline$\left[10^{-4}, 5 \times 10^{-4}\right]$ & DM3 & 1 & 387.30 \\
\hline$\left[5 \times 10^{-4}, 5.5 \times 10^{-4}\right]$ & DM5 & 2 & 238.90 \\
\hline$\left[5.5 \times 10^{-4}, 2.5 \times 10^{-3}\right]$ & DM3 & 2 & 193.50 \\
\hline$\left[2.5 \times 10^{-3}, 3 \times 10^{-3}\right]$ & DM5 & 3 & 159.30 \\
\hline$\left[3 \times 10^{-3}, 5 \times 10^{-3}\right]$ & DM3 & 3 & 129.00 \\
\hline$\left[5 \times 10^{-3}, 6.5 \times 10^{-3}\right]$ & DM3 & 4 & 96.80 \\
\hline$\left[6.5 \times 10^{-3}, 7 \times 10^{-3}\right]$ & DM3 & 5 & 77.40 \\
\hline$\left[7 \times 10^{-3}, 8 \times 10^{-3}\right]$ & DM3 & 6 & 64.50 \\
\hline$\left[8 \times 10^{-3}, 9.5 \times 10^{-3}\right]$ & DM3 & 7 & 48.40 \\
\hline$\left[9.5 \times 10^{-3}, 10^{-2}\right]$ & DM3 & 8 & 45.00 \\
\hline$\left[10^{-2}, 1.5 \times 10^{-2}\right]$ & DM1 & 4 & 27.20 \\
\hline$\left[1.5 \times 10^{-2}, 2 \times 10^{-2}\right]$ & DM1 & 5 & 21.76 \\
\hline$\left[2 \times 10^{-2}, 2.5 \times 10^{-2}\right]$ & DM1 & 7 & 15.54 \\
\hline$\left[2.5 \times 10^{-2}, 3 \times 10^{-2}\right]$ & DM1 & 9 & 12.10 \\
\hline
\end{tabular}

\section{Conclusion}

In this paper, we analyze the Bluetooth broadcasting scheme and provide results that can be used to improve broadcasting throughput and reliability. We used previously covered methods of reliability calculations in Bluetooth to come up with a novel method to create an adaptive packet type and repetition count $\left(N_{B C}\right)$ selection scheme. We observed that our scheme can successfully improve the performance of current Bluetooth broadcasting scheme (in terms of throughput and reliability) for various radio channel conditions.

\section{References}

1. Haarsten, J., Naghshineh, M., Inouye, J., Joeressen, O., Allen, W.: Bluetooth: Vision, Goals and Architecture, Mobile Computing and Communications Review, ACM SIGMOBILE, Vol. 2, No. 4 (1998)

2. Bluetooth Core Specification Version 1.1, Bluetooth SIG (1999)

3. Ronai, M.A.: Packet Level Simulation of Bluetooth Physical Layer, M.S. Thesis, Budapest University of Technology and Economics (2001)

4. Proakis, J., Salehi, M.: Communication Systems Engineering, Prentice Hall (2002)

5. Krassi, B.: Reliability of Bluetooth, 12th Conf. on Extreme Robotics, RTC, St. Petersburg (2001)

6. Chen, L-J.,Kapoor,R., Sanadidi,M.Y.,Gerla,M.:Enhancing Bluetooth TCP Throughput via Link Layer Packet Adaptation, In: Proceedings of IEEE ICC, France (2004) 